Research Article

\title{
AN OVERVIEW TO ANTENNA TERMINOLOGY AND DESIGN A COMPACT DUAL BAND ANTENNA BY USING HFSS PROGRAM FOR C AND X BANDS APPLICATIONS
}

\author{
Ali Recai ÇELIK ${ }^{1^{*}}$, Muhammed Bahaddin KURT ${ }^{2}$
}

Antennas are used to transform the traveling signals into the electromagnetic waves in the free space. There are many types of the antennas using in the various areas. Microstrip patch antennas have been used for many applications such as mobile communications, radar applications, microwave imaging, satellite systems, remote sensing, radio frequency identifications, wireless systems, synthetic aperture radar, telemetry, aerospace, weather mapping and detecting etc. These applications operate at $L, S, C, X$ and $K$ microwave bands or combinations of these bands. In this study, a comprehensive study of the antenna terminology is presented and a compact dual-band antenna operating at the C-band and Xband is developed and analyzed. Defected Ground Structure techniques are used on the ground side and some other modification techniques are used on the patch side in order to obtain the resonant frequencies in these two bands. The FR4-epoxy material is chosen as a substrate for the designed patch antenna with dielectric constant of 4.5, and height of $1.6 \mathrm{~mm}$. The antenna structure is optimized and simulated using High Frequency Structured Simulator program which uses the Finite Element Methods solver technique. A large number of the antenna parameters are obtained and explained at the result part of the study.

Key words: $C$ band, $X$ band, HFSS, Microstrip patch antenna

\section{Introduction}

Developments in the various areas of the radio frequencies and microwave technologies have been observed significantly over the last decades. Most of the applications of these areas operate at $\mathrm{S}$, $\mathrm{C}$ and $\mathrm{X}$ bands that cover 2-4 GHz, 4-8 GHz and 8-12 GHz, respectively [1]. Antennas have critical roles for many applications such as mobile communications, radar applications, microwave imaging, satellite systems, remote sensing, radio frequency identifications, wireless systems etc. An antenna needs to be optimized in order to be used in these specific applications. For this aim, several properties like bandwidth, size, gain, directivity, etc. must be adjusted to get the desired results. Among the various types of the antennas, microstrip patch antennas (MPAs) are suitable for using in many applications due to their compact size and low-profile structures. However, MPAs have the greatest disadvantage of their narrow band width [2].

\footnotetext{
${ }^{1}$ Department of Electrical and Electronics Engineering, University of Dicle, Diyarbakir, Turkey, (eeealicelik@gmail.com) (i) https://orcid.org/0000-0002-6917-5170

${ }^{2}$ Department of Electrical and Electronics Engineering, University of Dicle, Diyarbakir, Turkey, (bkurt@ dicle.edu.tr) (Dhttps://orcid.org/0000-0001-6983-2081 
In this paper, firstly the basic terminology of an antenna is explained. Then, information about the High Frequency Structural Simulator (HFSS) program which is widely utilized in the analysis of the electromagnetic structures [3] is given. Finally, a dual band antenna which operates at C-band and $\mathrm{X}$-band is designed by using defected ground structure (DGS) techniques on the ground side and some other modification techniques on the patch side. The modified MPA have slots on the ground and parasitic elements on the patch in order to obtain broad bandwidth in contrast to conventional MPAs. Design and analysis of the antenna is achieved by HFSS software.

\section{Important Antenna Parameters and HFSS Program}

In the development process of an antenna, it is necessary to keep various parameters in the specific values [4]. Some of the basic antenna parameters can be summarized as return loss $(R L)$, reflection coefficient $(\Gamma)$, antenna impedance $\left(Z_{A}\right)$, voltage standing wave ratio $(V S W R)$, bandwidth $(B W)$, gain $(G)$, directivity $(D)$, efficiency $(e)$ and half power beamwidth $(H P B W)$. In this section, these important antenna parameters are introduced and the relationships between them are explained with some equations.

\subsection{RL, $\Gamma, \mathrm{Z}_{0}$, VSWR, BW, G, D, $e$, Radiation Pattern and HPBW}

Both the antenna and the transmission cable which connecting the antenna to signal source should have same impedance values for transferring electromagnetic energy efficiently [5]. Transceivers and their transmission lines are generally have $50 \Omega$ impedance. If the antenna has $Z_{A}$ that is different from $50 \Omega$, then there is a mismatch and an impedance matching is required. The $R L$ is the other expression of the mismatch. It is a logarithmic ratio measured in decibel $(\mathrm{dB})$ that compares the power reflected by the antenna to the power that is fed into the antenna from the transmission line. There are significant relationships between the parameters of $R L, \Gamma, Z_{0}$, and VSWR as given below [2]:

$$
\begin{aligned}
& \Gamma=\frac{V_{R E F L}}{V_{I N C}}=\frac{Z_{A}-Z_{O}}{Z_{A}+Z_{O}} \\
& R L=-20 \log \Gamma \\
& V S W R=\frac{1+|\Gamma|}{1-|\Gamma|}
\end{aligned}
$$

where $V_{I N C}$ and $V_{R E F L}$ are incident and reflection voltage for the antenna feed point, respectively. $Z_{0}$ is the input impedance and generally equal to $50 \Omega$. According to the Equations (1-3), as the $R L$ falls below $-10 \mathrm{~dB}$ or $\Gamma$ falls below 1 or VSWR falls below 2, the impedance matching of the antenna will increased and the performance of the antenna will be good. Hence, one of the important purposes for an antenna designer is obtaining the mentioned values for $R L, \Gamma$ and $V S W R$.

Another basic antenna parameter is $B W$ which means the interval of the frequencies where the antenna can operate efficiently. In other words, $B W$ is the number of frequencies for $R L<-10 \mathrm{~dB}$ and $1<V S W R<2$. It can be formulated according to Eq. 4 [2].

$$
B W=2 \frac{f_{\max }+f_{\min }}{f_{\max }+f_{\min }} \%
$$

where $f_{\max }$ and $f_{\min }$ mean the maximum and minimum values of the frequency range, respectively. 
Directivity is the ability of an antenna to focus in a particular direction when transmitting or receiving energy. Another important parameter that expresses the performance of the antenna is Gain. There are significant relationships between the parameters of G, D and $e$ as given in Eq. 5 [6]:

$$
G=e \times D
$$

The relative strength of the radiated field at the different directions from the antenna is indicated by 'Radiation Pattern' which can be both two-dimensional and three-dimensional. The angular width, in which the power across the maximum radiation direction is halved, is defined as $H P B W$ and is a measure of the directionality. In decibels, half of the power is expressed as $3 \mathrm{~dB}$, for this reason the $H P B W$ is generally referred to $3 \mathrm{~dB}$ beamwidth. Two-dimensional and three-dimensional radiation patterns are given in Fig. 1 as an example [7]. The expression of the HPBW is also shown on this figure.

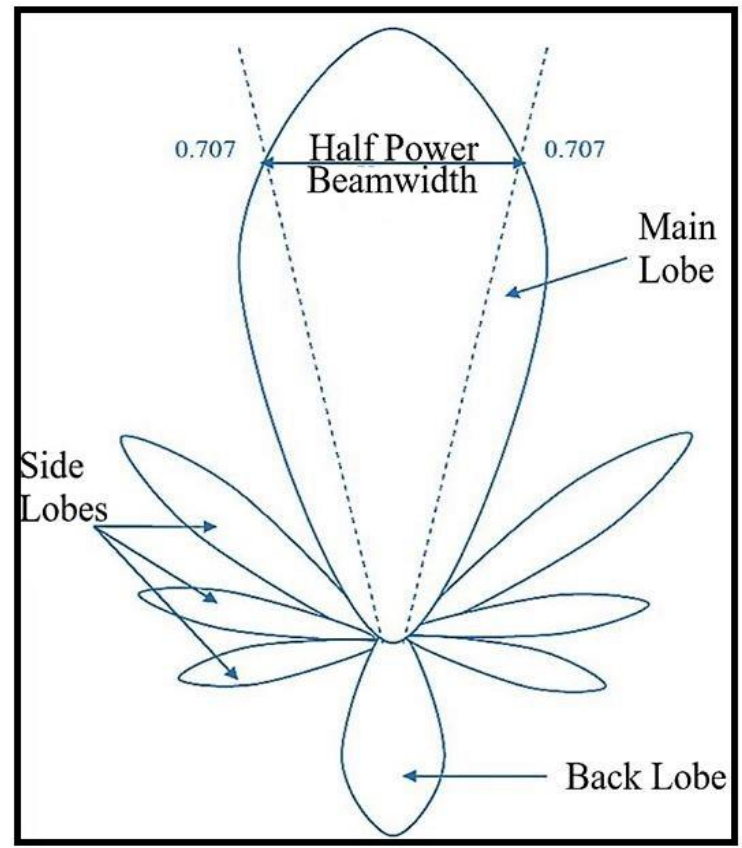

(a)

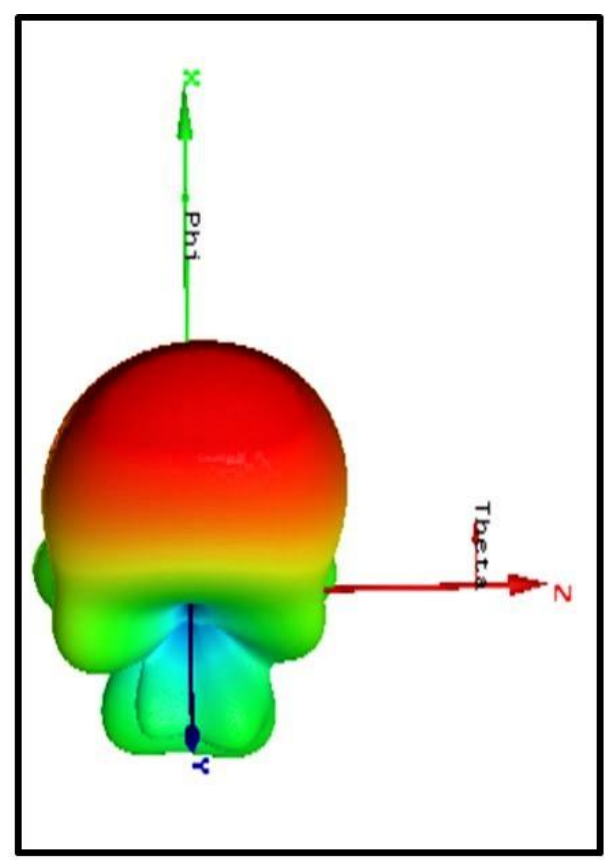

(b)

Figure 1. Radiation patterns: a) Two-dimensional, b) Three-dimensional.

\subsection{HFSS Program}

There are several antenna simulator programs to achieve the aims of designing parameters, modelling antennas and analysing the behaviours of them. These are generally classified into two numerical methods, namely Finite Difference Time Domain (FDTD) and Finite Element Method (FEM). The most known programs such as FEKO, Antenna Magus and HFSS use the FEM method that is a technique for finding approximate solutions to boundary value problems for differential equations [8]. Another important program Computer Simulation Technology (CST) Microwave Studio is based on the FDTD method [9].

In this study, the HFSS program is preferred to develop a compact dual-band antenna. HFSS is high performance electromagnetic field simulation software. Electromagnetic problems can be solved quickly and clearly by using it. Near and far electromagnetic fields can be drawn, parasitic parameters $(\mathrm{S}, \mathrm{Y}, \mathrm{Z}$ ) can be calculated and the antenna performance can be optimized by using this program. 


\section{Design and Analysis of Dual-Band MPA}

MPAs consist of a radiating patch, a ground plane and a dielectric substrate. They are generally fed by a microstrip line or a coaxial line. In this study, the microstrip feedline method which is easy to design and fabricate is used. An inset fed MPA is preferred to design due to certain planar shortages of the microstrip line feeding [10]. Firstly, design parameters of the antenna are calculated based on the transmission line model for obtaining desired resonant frequencies approximately. In the second stage of the development process, some modification such as using slots and parasitic elements are implemented to improve the performance of the antenna. Therefore, certain parameters are determined and the process is completed successfully. The transmission line model is given in Eq. 5 to 11 [6].

$$
\begin{aligned}
& W=\frac{c}{2 f_{0}\left[\frac{\left(\varepsilon_{r}+1\right)}{2}\right]^{1 / 2}} \\
& \varepsilon_{r_{e f f}}=\frac{\varepsilon_{r}+1}{2}+\frac{\varepsilon_{r}-1}{2}\left(1+\frac{12 h}{W}\right)^{-1 / 2} \\
& L_{e f f}=\left[\frac{c}{2 f_{0}\left(\varepsilon_{r_{e f f}}\right)^{1 / 2}}\right] \\
& \Delta L=0.412 h \frac{\left(\varepsilon_{r_{e f f}}+3\right)\left(\frac{W}{h}+0.264\right)}{\left(\varepsilon_{r_{e f f}}-0.258\right)\left(\frac{W}{h}+0.8\right)} \\
& L=L_{e f f}-\Delta L \\
& x_{0}=\frac{c}{\left(2 \varepsilon_{r_{e f f}}\right)^{1 / 2}}\left(\frac{4,65 \times 10^{-12}}{f_{0}}\right) \\
& y_{0}=\frac{L}{\pi} \cos ^{-1}\left(\frac{R_{\text {in }}}{R_{\text {in }}}\right)^{1 / 2}
\end{aligned}
$$

where $W$ and $L$ mean width and length of the rectangular patch, $x_{0}$ and $y_{0}$ mean gap and distance of the inset fed, respectively.

As mentioned before, for the developed antenna the dielectric substrate is selected as "FR4Epoxy" material which has the dielectric constant $\left(\varepsilon_{\mathrm{r}}\right)$ of 4.5 and height (h) of $1.6 \mathrm{~mm}$ [11]. Firstly, the resonance frequency of the antenna is desired to be around $7 \mathrm{GHz}$ (for $\mathrm{C}$ band). Hence, the transmission line model is used these values to calculate the antenna parameters. Input parameters and obtained output parameters are given in Table I. The top view and design parameters of the antenna are shown in Fig. 2.

Table 1. Obtained output parameters according to the inputs.

\begin{tabular}{|c|c|}
\hline Inputs & Outputs \\
\hline Dielectric Thickness $=1.6 \mathrm{~mm}$ & Patch Width $=15 \mathrm{~mm}$ \\
\hline Conductor Thickness $=0.035 \mathrm{~mm}$ & Patch Length $=13 \mathrm{~mm}$ \\
\hline Relative Permittivity $=4.5$ & Inset Gap $=2 \mathrm{~mm}$ \\
\hline Solution Frequency $=7 \mathrm{GHz}$ & Inset Distance $=5 \mathrm{~mm}$ \\
\hline Input Impedance $=50 \mathrm{Ohm}$ & Substrate Size $=42 \times 35 \mathrm{~mm}^{2}$ \\
\hline
\end{tabular}




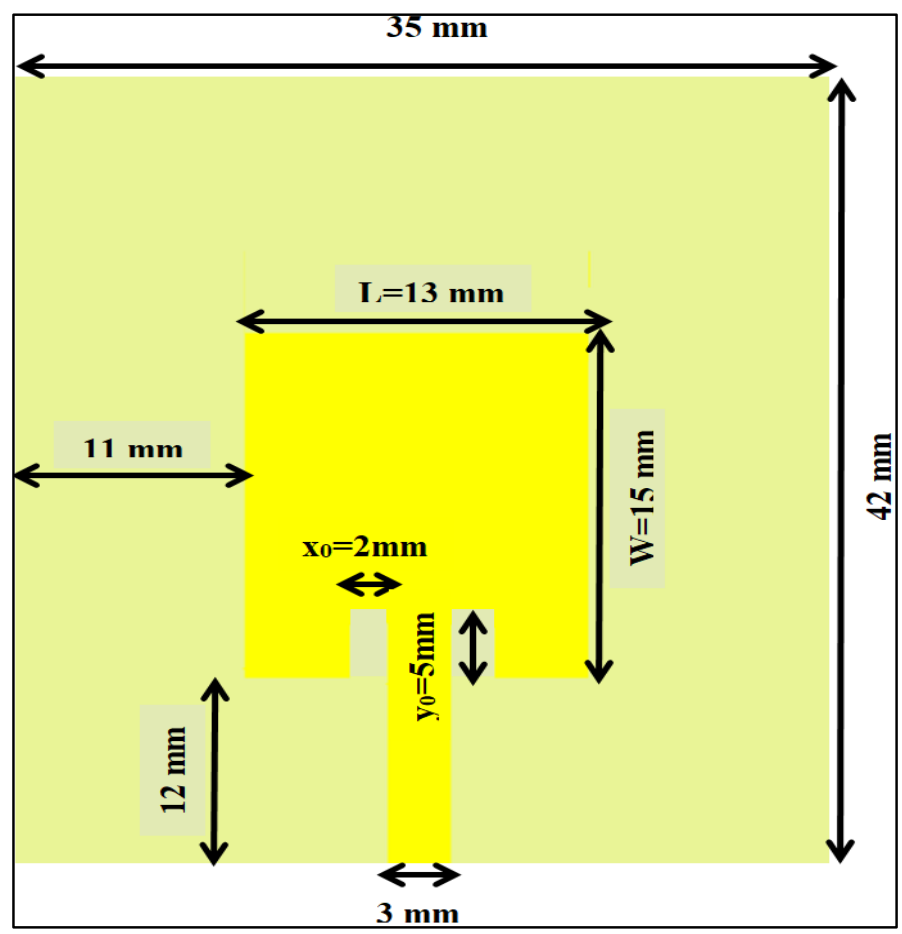

Figure 2. Designed inset-fed antenna to obtain $7 \mathrm{GHz}$ resonance frequency.

\subsection{Development Process and Modification Techniques}

The RL result of the designed antenna is given in Fig. 3. According to this plot, it is seen that the first step of the development process has been completed successfully. Between the $5.8 \mathrm{GHz}$ and 8.2 GHz, the RL values are under the $-10 \mathrm{~dB}$ level meaning the antenna is working efficiently. So, transmission line model has provided obtaining the $7 \mathrm{GHz}$ resonance frequency which is approximately in the C-band of the microwave spectrum. After this stage, the main purpose is obtaining new resonance frequency around the $10 \mathrm{GHz}$ for working in the X-band applications.

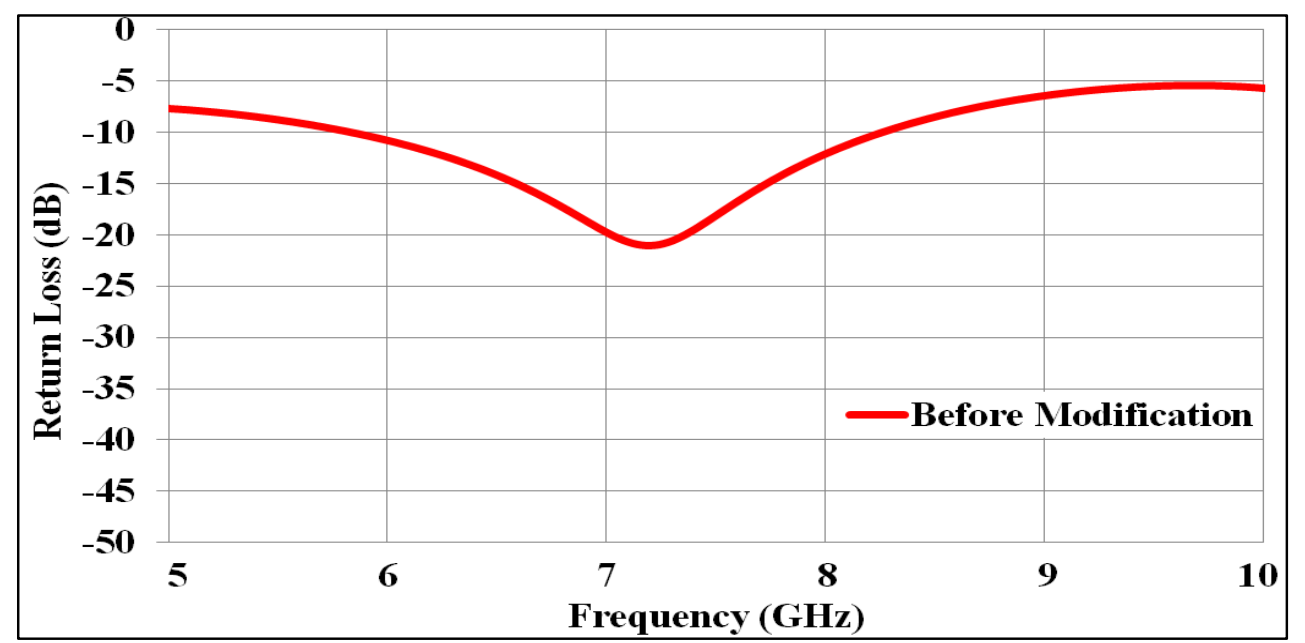

Figure 3. Return loss of the inset-fed antenna before implementing any modification techniques.

The new resonance frequencies can be occurred and BW of the antenna can be enhanced by using DGS approach $[12,13]$. The methods of etching slot(s) on the ground plane and adding parasitic element(s) on the patch side are the most used methods in order to improve the bandwidth of a compact MPA $[14,15]$. 
In this study, a new resonance frequency is obtained by using L-shaped and inverted L-shaped slots. The sizes and the positions of these slots are determined empirically in order to set the new frequency around the $10 \mathrm{GHz}$. Moreover, two parasitic elements having rectangular shape are added to reduce the RL values to lower levels.

The proposed antenna after making some modifications is shown in Fig. 4. After the modifications, the new RL result is obtained as seen in Fig. 5. According to this plot, the second resonance is occurred between the $9.6 \mathrm{GHZ}$ and $11.2 \mathrm{GHz}$ that is in the X-band of the microwave spectrum. Furthermore, the RL level approaches $-50 \mathrm{~dB}$ values that provides one of the purposes of this work. Even if the first resonance frequency shifted to left, it has still stayed at the desired range for C-band. Also, the 3-D plot of the gain values is shown in Fig. 6. It is clearly seen from the figure that the proposed antenna has omnidirectional radiation pattern meaning the antenna emits uniform wave power in all directions in a plane.

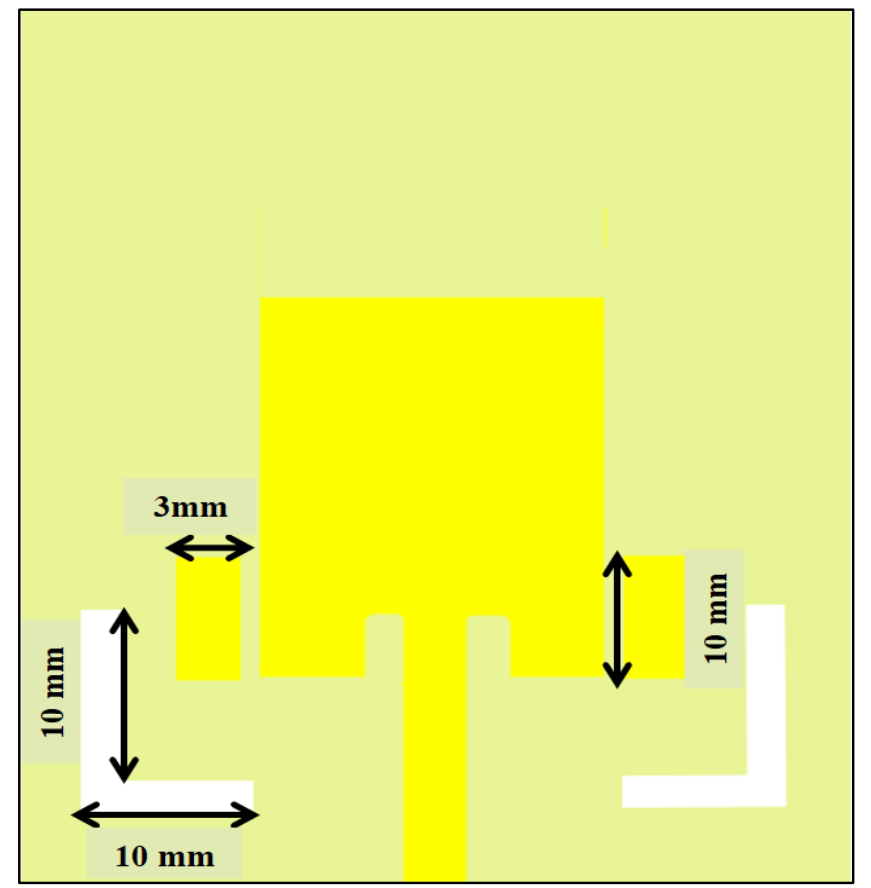

Figure 4. Proposed antenna after making some modifications.

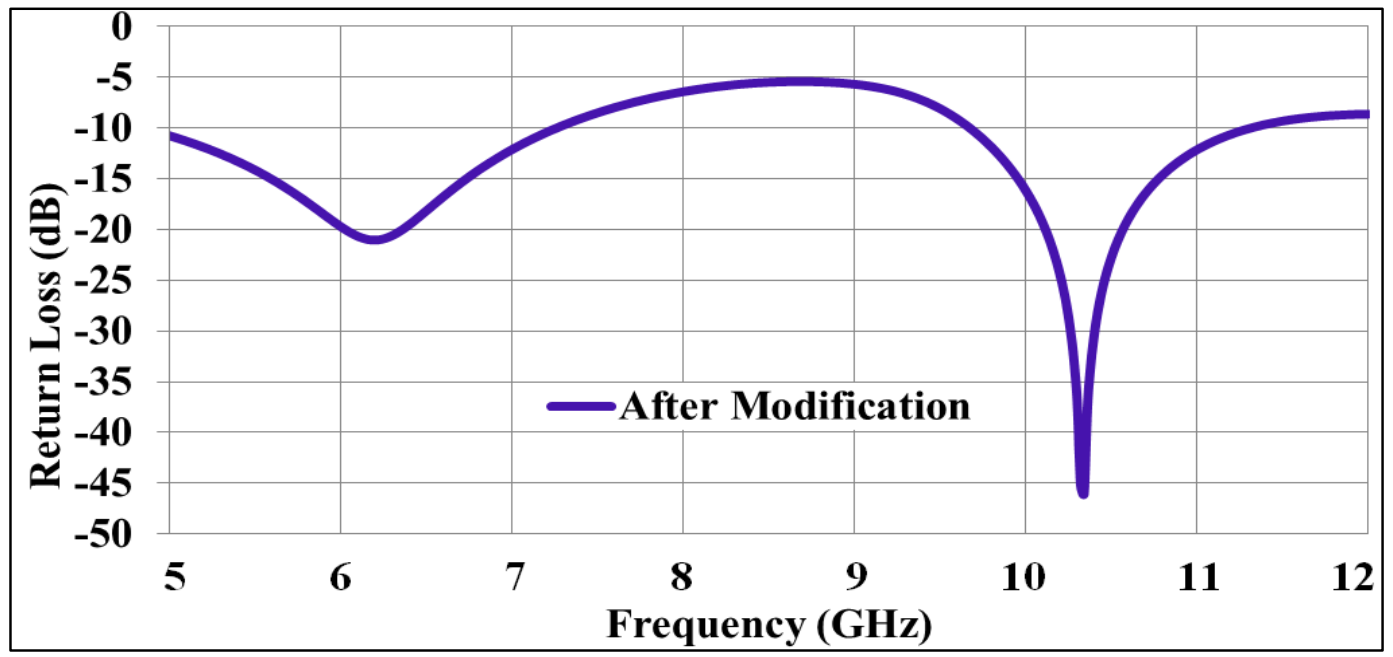

Figure 5. Return loss of the antenna after implementing some modification techniques. 


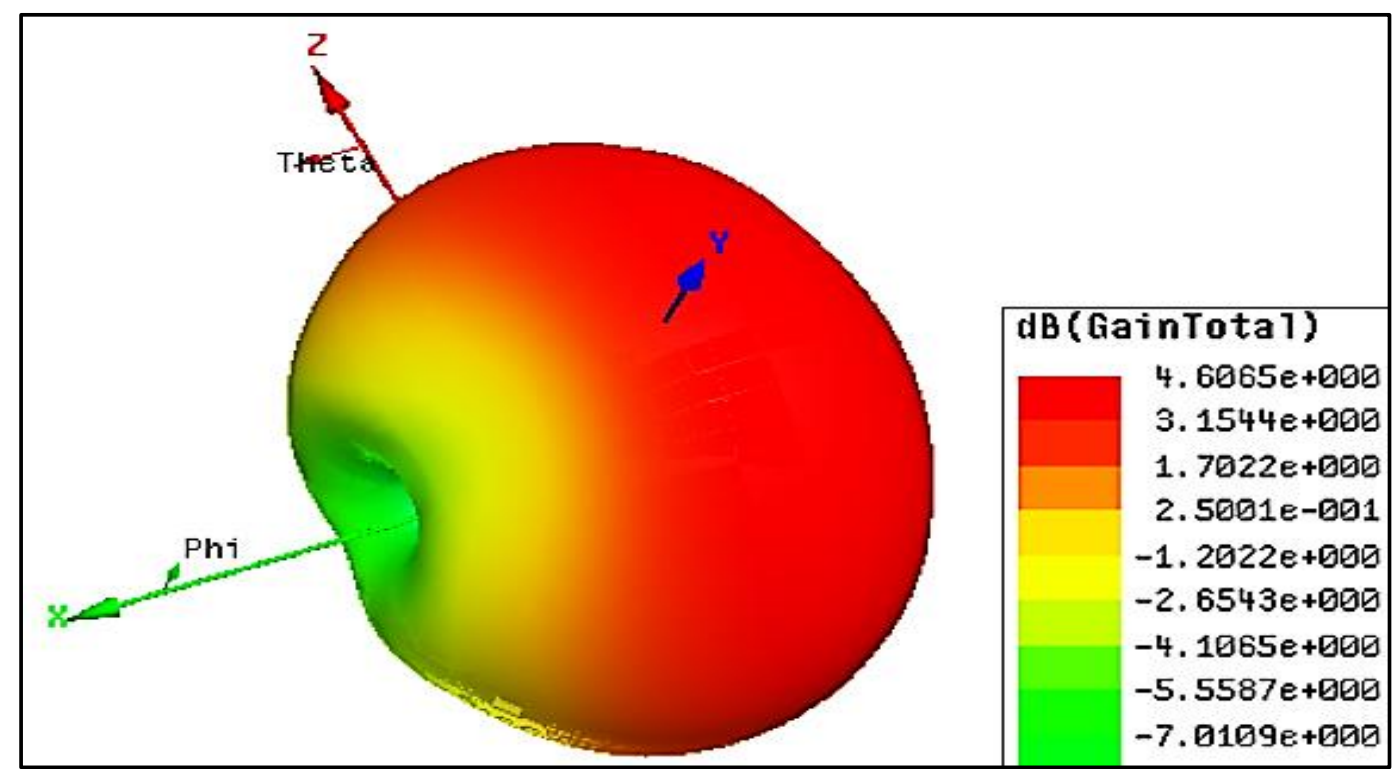

Figure 6. 3-D polar plot of the gain.

\section{Conclusions}

The various types of the antennas with different properties are used in several applications. The performance of an antenna is related to impedance matching, bandwidth, gain, efficiency, beamwidth and size of the antenna. Optimization of these parameters is continued by researchers so as to obtain better analysis results. For this purpose, the simulation programs and their tools play a critical role in the development process of the antenna. In this paper, a comprehensive study of the antenna terminology is presented and HFSS simulation program is introduced. In the simulation study, a compact $\left(42 \times 35 \times 1.6 \mathrm{~mm}^{3}\right)$ microstrip dual-band antenna operating at the two broad frequency bands that make it suitable for $\mathrm{C}$ and $\mathrm{X}$-bands applications is proposed. This antenna has FR-4 Epoxy substrate and inset-fed rectangular patch. DGS and trial-error optimization technique are used to improve the return loss performance of the antenna. Two slots are etched on the ground plane and two parasitic elements are added to the patch side of the antenna. After the parametric analysis, the bandwidths of $34 \%(5.8-8.2 \mathrm{GHz})$ and $15 \%(9.6-11.2 \mathrm{GHz})$ are obtained in the C-band frequency range $(4-8 \mathrm{GHz})$ and $\mathrm{X}$-band frequency range $(8-12 \mathrm{GHz})$, respectively. The proposed antenna is applicable for various systems working in the C-band such as satellite communication, weather radar systems, Wi-Fi and ISM Band applications, and for various systems working in the X-band such as radar applications and space communications. Some other modification techniques can be implemented to obtain whole frequency range of $\mathrm{C}$ and $\mathrm{X}$ bands. It is considered that, the proposed antenna will be ready to fabrication process after some improvements.

\section{Acknowledgment}

This paper is the extended version of a conference paper which was presented orally in the International Engineering and Natural Sciences Conference (IENSC'18). The research is supported by the Researching Projects Committee of the University of Dicle (DUBAPK) with the project number of 14-MF-71. We are grateful to DUBAPK for financial assistance. 


\section{References}

[1] Islam, M. F., et al., Dual band microstrip patch antenna for SAR applications. Australian Journal of Basic and Applied Sciences, 4(2010), 4585-4591.

[2] Balanis, C. A., Antenna Theory: Analysis and Design, John Wiley and Sons Inc., New Jersey, USA, 2015.

[3] Ansys Hfss (ver. 15), Ansys Corporation. Canonsburg, PA, USA. Retrieved from http://www.ansys.com/Products/Electronics/ANSYS-HFSS, 2014.

[4] Celik A.R., Kurt, M.B., Development of a novel ultra-wideband, stable and high directive monopole disc antenna for radar-based microwave imaging of breast cancer, Journal of Microwave Power and Electromagnetic Energy, 52(2), 75-93.

[5] Kraus, J.D., Antennas, McGraw Hill, New York, USA, 1988.

[6] Ulaby, F., 2006. Fundamentals of Applied Electromagnetics, 5th edition, Prentice Hall, Washington DC. USA, 2006.

[7] Celik, A.R., Research \& Reviews in Engineering, Gece Kitapligi, Ankara, Turkey, 2019.

[8] Bardi, I., Cendes, Z., New directions in HFSS for designing microwave devices, Microwave Journal, 41(8), 22-36.

[9] Computer Simulation Technology, http://www.cst.com

[10] Garg, R., et al., Microstrip Antenna Design Handbook, Artech House Antennas and Propagation Library, 2001

[11] FR4 Epoxy Properties, Available: https://www.microwaves101.com/encyclopedias/fr-4

[12] Guha, D., et al., Microstrip patch antenna with defected ground structure for cross polarization suppression, IEEE Antennas and Wireless Propagation Letters, 4(2005), 455-458.

[13] Dong, J., et al., Compact planar ultrawideband antennas with 3.5/5.2/5.8 GHz triple bandnotched characteristics for internet of things applications, Sensors, 17(2017).

[14] Bah, J., et al., Ground slotted monopole antenna design for microwave breast cancer detection based on time reversal MUSIC, Progress In Electromagnetics Research C, 59(2015), 117-126.

[15] Wani, Z., Kumar, D., Dual-band-notched antenna for UWB MIMO applications, International Journal of Microwave and Wireless Technologies, 35(2015), 1-6. 